\title{
Sex determination by the interarticular distance of metacarpals and phalanges: a digital radiologic study in contemporary Turkish people
}

\author{
Banu Alıcıŏ̆lu' ${ }^{1}$ Ali Yılmaz ${ }^{2}$, H. Muammer Karakaşs ${ }^{3}$ Bülent Sabri Cigalı, \\ Selman Çıkmaz ${ }^{2}$, Enis Uluçam² \\ ${ }^{1}$ Department of Radiology, Medical Faculty, Trakya University, Edirne, Turkey \\ ${ }^{2}$ Department of Anatomy, Medical Faculty, Trakya University, Edirne, Turkey \\ ${ }^{3}$ Department of Radiology, Medical Faculty, İnönii University, Turgut Özal Medical Center, Malatya, Turkey
}

\begin{abstract}
Objectives: The ability to determine sex from isolated bones and bone fragments is a necessity in medicolegal investigations. While the pelvis and cranium are the most useful bones for these determinations, they are not always available. The purpose of the present work is to obtain a discriminant equation for the metacarpals and phalanges in a live Turkish population.

Methods: Interarticular distances of the metacarpals and phalanges of the left hands of 22 males and 43 females were measured on digital roentgenograms. Means, standard deviations and minimum and maximum values were found.

Results: A multivariate logistic regression model was formed to estimate sex, with $72.7 \%$ of the males, $90.7 \%$ of the females and $84.6 \%$ of the pooled individuals being correctly classified when the cut value was 0.5 .

Conclusion: We consider that the length of metacarpals and phalanges are potential bones that can be used for sex determination in forensic science. Radiologic measurements are an optimal alternative to population studies where the number of well-protected cadavers is limited or unavailable.
\end{abstract}

Key words: digital radiology; finger phalanges; metacarpal bones; sex determination

Anatomy 2009; 3: 14-20, (C) 2009 TSACA

\section{Introduction}

Forensic anthropologists are continually attempting to improve methods of skeletal identification through the development of new methods for determining sex or the fine-tuning of known methods on various parts of the skeleton. ${ }^{1}$ Although only two biological sexes exist in humans, sex estimation of a human skeleton is a very complex diagnosis. For this purpose, many studies that use the pelvis and skull have been conducted on different populations from all over the world on skeletal remnants obtained from skeletal collections or exhumed graves ${ }^{2,3}$ However, when skeletal remains are incomplete or damaged, the sex determination must be attempted from other skeletal bones because most of the other bones show some degree of sexual dimorphism. ${ }^{4-6}$ In the same way, metacarpals and phalanges shows dimorphism by means of osteometric and geometric features, which is explained by the fact that men and women do different things, to different degrees. ${ }^{7-9}$ Likewise, it is known that 
the human male mean skeletal dimensions generally exceed those of females. Previous studies have shown that the metacarpals are useful for the sex determination of skeletal remnants, but they obtained conflicting results in terms of accuracies that were explained by racial, temporary or populational variances. ${ }^{4-13}$ There have been fewer studies on phalanges for the same purpose, although their accuracy has been found to be higher than that of metacarpals. ${ }^{7}$ Anatomically short tubular bones have some advantages over other bones in a forensic context. The shafts of long bones often stay intact, but their epiphyses are prone to damage because of the overlying fragile cancellous bone. However, the smaller long bones of the hands often remain complete. ${ }^{4}$

The aim of this study is to determine the interarticular distances of both metacarpals and phalanges on digital roentgenograms and to develop a discriminant function formula that can be used to estimate sex. The datasets of the left hand were used because of the fact that the majority of the population is right-handed and therefore will be less influenced by activity.

\section{Materials and Methods}

\section{Study subjects and radiologic evaluation}

Dorso-volar roentgenograms of the left hand were studied cross-sectionally in 65 adult patients consisting of 22 males (33.8\%) and 43 females (66.2\%) in the Trakya University Radiology department. The films were examined by an experienced musculoskeletal radiologist and an anatomy Ph.D. student (first author) on a general-purpose Polystar Siemens workstation. The cases with skeletal immaturity, pathological lesions such as congenital and developmental dysplasia, metabolic bone diseases, recent trauma or surgery, as well as tumors, osteoarthritis and arthritis were excluded.

In order to have more accurate measurements and to better clarify the anatomical structures, the scans were enlarged. The interarticular distance was measured as the distance between the midpoint of the basis and distal tip point of all the metacarpals and phalanges (proximal, middle and distal) by electronic calipers (Figure 1).

Figure 1. The measurements of the metacarpals and phalanges on dorso-volar hand roentgenogram. S (Space) 1: first distal phalange; S2: first proximal phalange; S3: first metacarpal, etc.

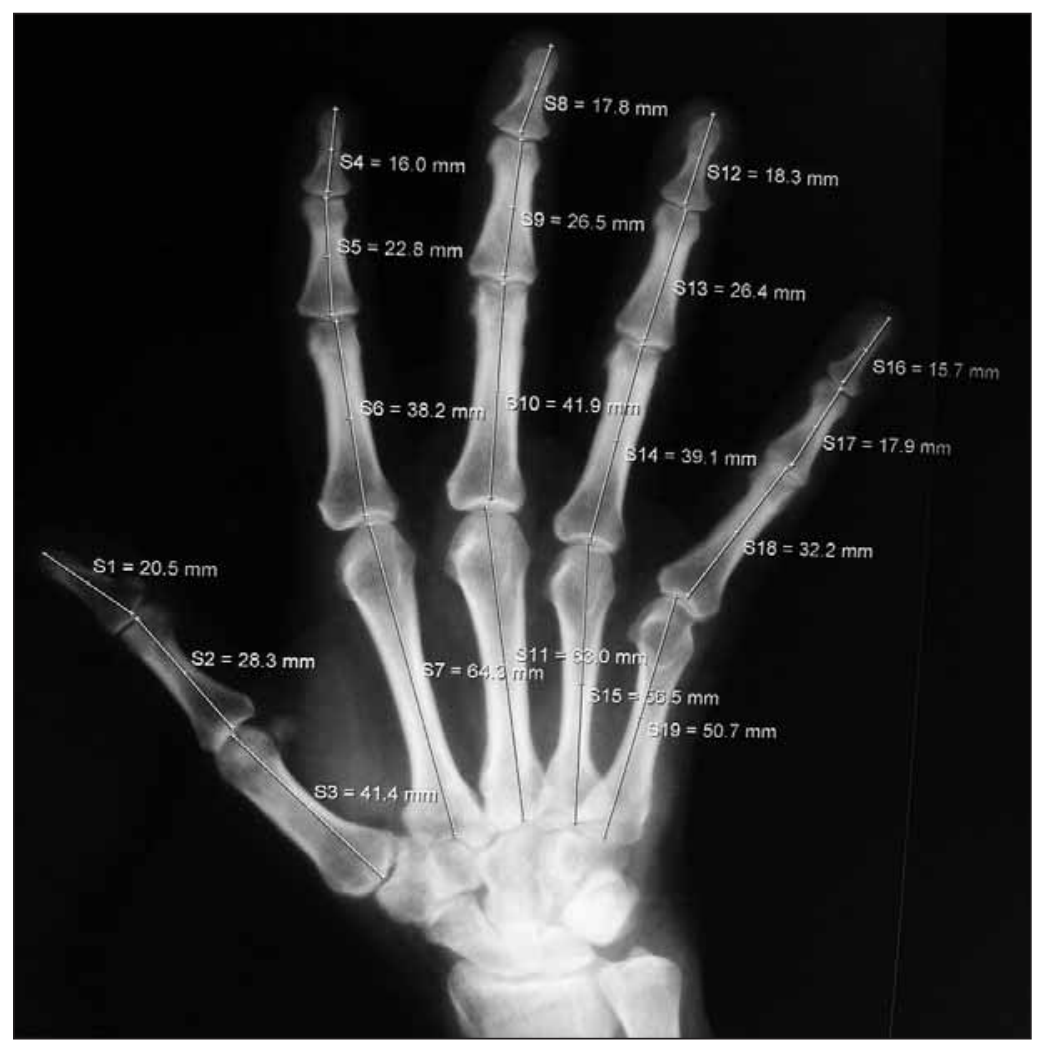




\section{Statistical analyses}

All results were expressed as mean \pm standard deviation (SD), minimum and maximum values.

Because of the dichotomous nature of the sex type, logistic regression was used in order to be able to predict sex based on values of predictor variables defined as the interarticular distance of the metacarpals and phalanges. A statistical model was estimated using block entry of all variables and a statistically determined constant.

A p-value of $<0.05$ was considered as statistically significant. STATISTICA 15.0 was used for statistical analyses.

\section{Results}

Table 1 summarizes the descriptive analyses by means of age of the subjects. Figure 2 shows the age distribution of the subjects.

Table 1

Descriptive statistics of the subjects by means of age

\begin{tabular}{lcccc}
\hline & Minimum & Maximum & Mean & SD \\
\hline Male $(n=22)$ & 18 & 74 & 40.5 & 16.7 \\
Female $(n=43)$ & 18 & 75 & 49.6 & 14.6 \\
Total $(n=65)$ & 18 & 75 & 46.5 & 15.9 \\
\hline
\end{tabular}

The summary statistics for all the measurements are presented in Table 2.

A multivariate logistic regression model was formed to estimate sex using the interarticular distances of all the metacarpals and phalanges of the left hand. Sex was determined as the dependent variable; the results are presented in Table 3.

\section{Statistical formula}

$$
\begin{aligned}
& 1 /\left(1+e^{-z}\right) \\
& e=2.718
\end{aligned}
$$

Sex (z), where $\mathrm{z}=1$ for males and $\mathrm{z}=0$ for females, was regressed on the bone measurements (DP1, PP1, $\mathrm{MC} 1, \ldots)$ to produce equations with the generalized formula.

$$
\begin{aligned}
& \mathrm{z}=\beta_{0}+\beta_{1} \chi_{1}+\beta_{2} \chi_{2}+\ldots \ldots+\beta_{\mathrm{k}} \chi_{\mathrm{k}} \\
& \beta_{0}=\text { intercept } ; \beta_{1}, \beta_{2}, \beta_{3}, \ldots=\text { regression ceofficients }
\end{aligned}
$$
of $\chi_{1}, \chi_{2}, \chi_{3}$ respectively

$$
\mathrm{z}=-28.919+2.154(\mathrm{DP} 1)+2.074(\mathrm{PP} 1)-0.165
$$

$(\mathrm{MC} 1) \ldots$

If $\mathrm{z}>0.5$, the bone is male, and, if $\mathrm{z}<0.5$, the bone is female.

Regression formula $=1 /\left(1+2.718^{-\mathrm{z}}\right)$

As a result, $72.7 \%$ of the males (16/22), $90.7 \%$ $(39 / 43)$ of the females and $84.6 \%$ of the pooled individuals were correctly classified when the cut value was 0.5 .

\section{Discussion}

Several previous studies have addressed the issue of hand bones for their sex estimation potential, with most focusing on metacarpals. ${ }^{4,8,9,11}$ Scheuer and Elkingto ${ }^{4}$ developed regression equations by using six measurements (interarticular length, maximum midshaft diameter, antero-posterior and medio-lateral base breadths) of all metacarpals and the first phalanx in a sample of 60 fresh bones of British Whites. They obtained 74\% to $94 \%$ accuracy in the correct determination of sex, and the first metacarpal was found to give the highest degree of accuracy in identifying sex.

Falsetti ${ }^{11}$ analyzed three samples (212 individuals from the Terry Collection -consisting of EuropeanAmericans and African-Americans both Black and White - and 33 individuals from the Royal Medical School, UK. He generated linear discriminant functions based on five measurements of five metacarpals and tested populational differences and found significant metric differences for digits 1 and 3 . He concluded that the functions of MC2, MC4 and MC5 could provide an alternative method for sex assessment. Using these metacarpals, the correct classification rate was between $84.4 \%$ and $92 \%$. Smith ${ }^{10}$ used individuals from the Terry and Huntington-Canadians-Collections. He used stepwise discriminant analysis and reached an accuracy of between $87 \%$ and $98 \%$ for the correct assessment of sex using measurements of metacarpals and proximal, middle and distal phalanges. With the selection of models, between $70 \%$ and $90 \%$ of individuals were correctly classified. 
Table 2

Summary statistics (Minimum-maximum, mean and standart deviation) in milimeters, for males and females

\begin{tabular}{|c|c|c|c|c|c|c|}
\hline & Bone & 1st finger & 2nd finger & 3rd finger & 4th finger & 5th finger \\
\hline \multirow{8}{*}{ Male } & \multirow{2}{*}{ DP } & $16.4-24.8$ & $12.2-18.5$ & $12.8-20.2$ & $14.1-21.8$ & $11.9-17.9$ \\
\hline & & $20.5 \pm 2.2$ & $15.8 \pm 1.6$ & $17.0 \pm 1.9$ & $16.8 \pm 1.4$ & $14.9 \pm 1.6$ \\
\hline & \multirow{2}{*}{ MP } & \multirow{2}{*}{ None } & $15.9-26.5$ & $20.0-34.0$ & $19.1-30.5$ & $10.3-21.8$ \\
\hline & & & $22.1 \pm 2.5$ & $26.8 \pm 3.0$ & $24.5 \pm 1.7$ & $17.23 \pm 2.4$ \\
\hline & \multirow{2}{*}{ PP } & $21.1-33.5$ & $29.6-43.6$ & $33.7-49.1$ & $31.4-45.7$ & $24.1-35.1$ \\
\hline & & $29.0 \pm 2.3$ & $37.9 \pm 3.6$ & $42.6 \pm 4.0$ & $37.8 \pm 2.4$ & $30.9 \pm 3.1$ \\
\hline & \multirow{2}{*}{ MC } & $33.6-51.5$ & $51.0-77.0$ & $49.1-71.7$ & $41.7-64.5$ & $38.2-62.3$ \\
\hline & & $43.9 \pm 4.7$ & $65.2 \pm 7.0$ & $62.3 \pm 6.3$ & $55.6 \pm 3.5$ & $51.8 \pm 5.6$ \\
\hline \multirow{8}{*}{ Female } & \multirow{2}{*}{ DP } & $15.7-22.7$ & $11.3-19.3$ & $12.4-19.0$ & $13.5-19.8$ & $13.5-19.8$ \\
\hline & & $18.9 \pm 1.7$ & $15.4 \pm 1.7$ & $16.4 \pm 1.6$ & $16.8 \pm 1.4$ & $16.8 \pm 1.4$ \\
\hline & \multirow{2}{*}{ MP } & \multirow{2}{*}{ None } & $18.1-24.8$ & $22.0-30.0$ & $21.5-28.6$ & $12.4-20.0$ \\
\hline & & & $21.0 \pm 1.7$ & $25.8 \pm 2.0$ & $24.5 \pm 1.7$ & $16.7 \pm 2.0$ \\
\hline & \multirow{2}{*}{ PP } & $23.6-31.5$ & $32.4-42.0$ & $35.7-47.3$ & $34.3-43.4$ & $26.4-35.3$ \\
\hline & & $27.4 \pm 1.9$ & $36.5 \pm 2.4$ & $40.5 \pm 2.7$ & $37.8 \pm 2.4$ & $29.7 \pm 2.2$ \\
\hline & \multirow{2}{*}{ MC } & $36.6-48.5$ & $54.0-72.0$ & $53.4-68.8$ & $47.5-62.6$ & $41.7-57.4$ \\
\hline & & $42 \pm 3.0$ & $62.8 \pm 4.0$ & $60.5 \pm 3.5$ & $53.6 \pm 3.5$ & $50.0 \pm 3.4$ \\
\hline
\end{tabular}

DP: distal phalange; MP: middle phalange; PP: proximal phalange; MC: metacarp

Stojanovski $^{9}$ analyzed 200 individuals of the 30 European-American females and 15 AfricanUniversity of New Mexico Collection including 55 American females. Sex prediction accuracy ranged from European-American males, 22 African-American males, $79 \%$ to $85 \%$ with the use of 35 different linear discrim-

Figure 2. Age distribution of the study subjects.

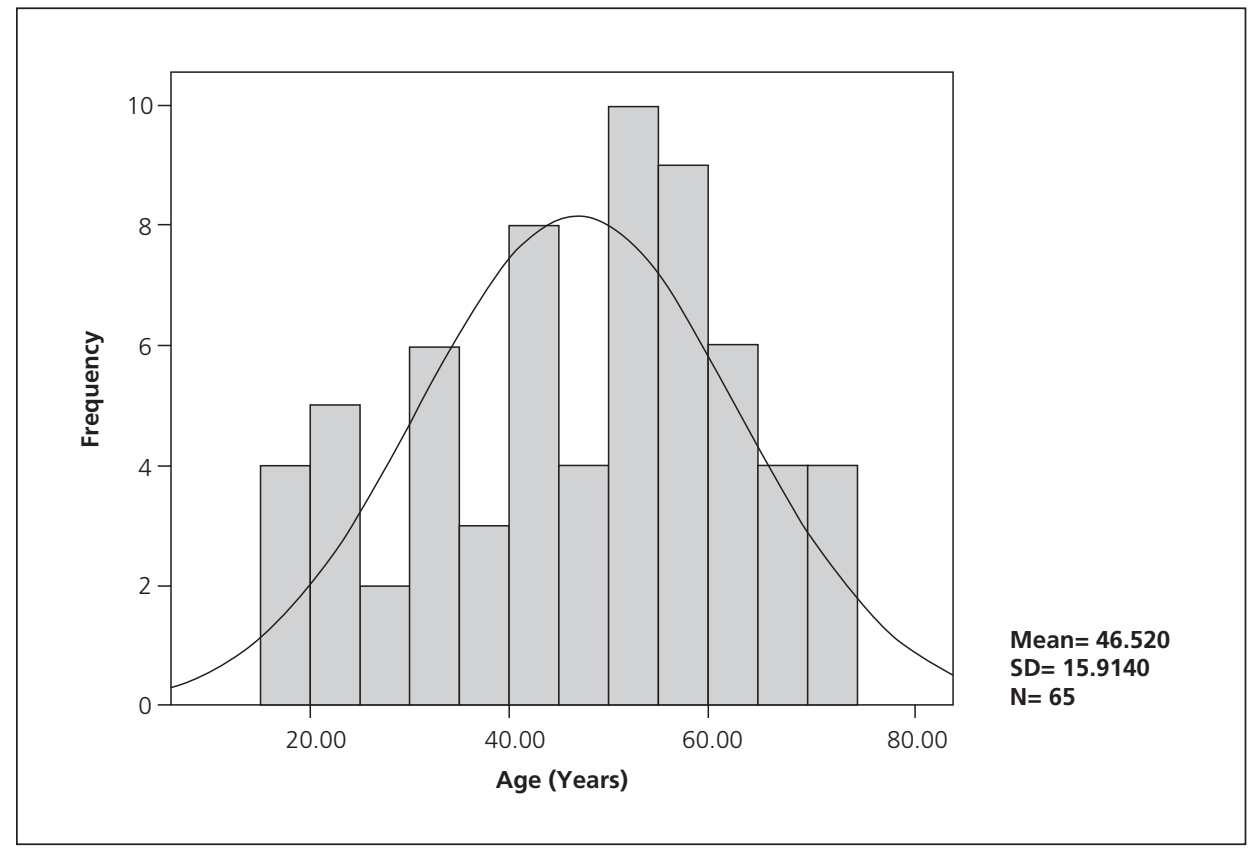


Table 3

The results of the multivariate logistic regression variation analyses with the use of the interarticular distances of the metacarpals and phalanges of the left hand as independent variables

\begin{tabular}{llrrrrrr}
\hline Variables & & B & Standart Error & Wald & df & Significance & Exp(B) \\
\hline Step 1(a) & DP1 & 2.154 & 1.256 & 2.939 & 1 & .086 & 8.620 \\
& PP1 & 2.074 & 1.091 & 3.609 & 1 & .057 & 7.953 \\
& MC1 & -.165 & .362 & .208 & 1 & .649 & .848 \\
& DP2 & -2.865 & 1.857 & 2.379 & 1 & .123 & .057 \\
MP2 & .012 & .888 & .000 & 1 & .989 & 1.012 \\
PP2 & -1.567 & 1.021 & 2.354 & 1 & .125 & .209 \\
MC2 & -.810 & .548 & 2.182 & 1 & .140 & .445 \\
DP3 & -2.190 & 1.393 & 2.471 & 1 & .116 & .112 \\
MP3 & 1.957 & 1.433 & 1.864 & 1 & .172 & 7.075 \\
PP3 & 1.781 & 1.433 & 1.544 & 1 & .214 & 5.934 \\
MC3 & .608 & .409 & 2.212 & 1 & .137 & 1.837 \\
DP4 & -1.257 & 1.009 & 1.552 & 1 & .213 & .285 \\
MP4 & -2.358 & 1.638 & 2.072 & 1 & .150 & .095 \\
PP4 & 2.045 & .958 & 4.556 & 1 & .033 & 7.731 \\
MC4 & -1.481 & .867 & 2.921 & 1 & .087 & .227 \\
DP5 & 4.616 & 2.284 & 4.086 & 1 & .043 & 101.123 \\
MP5 & -.833 & .490 & 2.885 & 1 & .089 & .435 \\
PP5 & -1.192 & 1.042 & 1.307 & 1 & .253 & .304 \\
MC5 & .482 & .424 & 1.296 & 1 & .255 & .000 \\
Constant & -28.919 & 13.476 & 4.605 & 1 & .032 & 1.620 \\
\hline
\end{tabular}

SE: standart error; Wald: $\chi^{2}$; df: degree of freedom; p: significance; Exp: exponantial.

ination functions. He utilized between two and five variables for each discriminatory function. MC4 produced the most consistently accurate estimates. That is, he found the most consistent accuracy using functions derived from MC4, and the lowest consistency with MC2. Burrows et al. ${ }^{6}$ tested the validity of each methodology described by Falsetti, Stojanowski and Scheuer and Elkington in 23 adult White Euro-American cadavers consisting of 11 females and 12 males. The ranges of accuracy obtained were lower than those reported by the original investigators except for in the results obtained using Stojanowski's methods. ${ }^{9}$ The accuracy range obtained using the methods of Scheuer and Elkington ${ }^{4}$ was $63.04 \%$ to $91.1 \%$ with MC2 producing the greatest accuracy and MC3 the lowest accuracy. In analyses that used Falsetti's method, ${ }^{11}$ pooled accuracy ranged between $83.3 \%$ and $87.0 \%$, with the greatest accuracy attained from MC2 and the lowest from MC4. Using Stojanowski's methods,' pooled accuracy was between $65.2 \%$ and $95.7 \%$, with the most consistent accuracy being obtained with functions derived from MC2 and MC3 and the lowest accuracy noted with MC5. Females were underperformed in the test sample by between $4.5 \%$ and $15.7 \%$ because of their low numbers.

Barrio et al. ${ }^{12}$ obtained 120 discriminant functions for each metacarpal from both hands by the measurement of eight parameters in 79 adult Spanish contemporary individuals. They reached $91 \%$ accuracy for left MC2 and $81 \%$ for right MC4 and MC5. 
The aforementioned studies, ${ }^{4,8,11}$ considered five or six measurements of the metacarpals and subsequent generation of regression equations or discriminant functions using the variables. Lazenby ${ }^{8}$ stated that the widths of metacarpals are more sexually dimorphic than their lengths; nevertheless, length is still a significant factor in sex discrimination. On the other hand, Case and Rose ${ }^{7}$ proposed that the measurements of mid-diaphyseal diameter, base, width and so forth reflects the robustness of the long bones and are therefore affected by activity. Consequently, they can disguise underlying patterns of sexual dimorphism. The measurements of the base and head of the metacarpals or phalanges continue to change after puberty through appositional growth. Therefore, modeling the assessment of sex by utilizing the three measurements increases the risk of errors. This thesis is supported by Ruff's study ${ }^{13}$ that concluded the diaphyseal cross-sectional size of the femur changes with mechanical loading. Our design focused on one measurement because the other measurements (antero-posterior width of base and head) of the metacarpals and phalanges are not accurate on routine hand radiographs. In addition, we restricted the study to left hands since the left side is less influenced by activity. Previous studies have shown that the accuracy rates for the left hand are higher. ${ }^{7,10}$ According to Lazenby, ${ }^{5}$ the effect of side asymmetry is controversial in industrial populations.

Case and Rose ${ }^{7}$ investigated the use of the length of metacarpals and phalanges for sex determination in 259 individuals (123 females, 136 males) from the Terry Anatomical Collection consisting of White Americans of European descent and European immigrants. The methodology of this study is the most comparable to ours; however, they measured the total length of the metacarpals and phalanges whereas we measured interarticular distance. Their results showed that the phalanges were better than the metacarpals for estimating sex and that the ability of the phalanges to correctly determine sex seems to improve in a distal direction. For the right hand, Case and Rose achieved $84.3 \%$ accuracy with the use of distal phalanges, $82.7 \%$ with the use of proximal phalanges, $77.9 \%$ with the use of both metacarpals and middle phalanges and $83.1 \%$ with the use of all metacarpals and phalanges. For the left hand, they achieved $85.7 \%$ accuracy with the use of distal phalanges, $80.8 \%$ with the use of middle phalanges, $80.8 \%$ with the use of proximal phalanges, $79.6 \%$ with the use of metacarpals and $82.9 \%$ with the use of all metacarpals and phalanges.

The accuracy rates of our study are comparable to those of previous studies. With the use of all metacarpals and phalanges, $72.7 \%$ of the males, $90.7 \%$ of the females and $84.6 \%$ of the total individuals were classified correctly. The lower accuracy rate of the males resulted from the fewer number of males in our study.

The results of the previous reports reflect secular, racial and social variances. ${ }^{11}$ The regression equations described should be applied cautiously for different populations and time periods. ${ }^{6}$ It seems likely that the previous studies conducted using older collections may not be appropriate because of secular alterations in metacarpal dimensions. Heterogeneous populations of the collections and the limited number skeletons -in particular, the minority of females - could be similarly problematic. In this context, digital radiologic studies offer researchers a visualization of the skeleton and precise metric analyses. Additionally, radiologic studies enable live persons to be imaged; therefore, widespread populations may be studied noninvasively, and the contemporary datasets of the populations may be obtained. Finally, they allow us to store large amounts of data electronically, which can be re-interpreted at any time. ${ }^{14}$

To summarize, this study reports the anthropometric data of the interarticular distance of metacarpals and phalanges of contemporary Turkish people and presents a mathematical model that allows sex determination. We propose that these discriminant functions could alternatively be used in forensic cases especially when the skull and pelvis are not available, and, given that all metacarpals and phalanges are available, the determination of sex is accurate. 


\section{References}

1. Steyn M, İşcan A. Metric sex determination from the pelvis in modern Greeks. Forensic Sci Int 2008; 179: 86.e1-6.

2. Rösing FW, Graw M, Marré B, et al. Recommendations for the forensic diagnosis of sex and age from skeletons. Homo 2007; 58 : 75-89.

3. Bruzek J. A method for visual determination of sex, using the human hip bone. Am 7 Phys Anthropol 2002; 117: 157-68.

4. Shreuer JL, Elkington NM. Sex determination from metacarpals and the first proximal phalanx. F Forensic Sci 1993; 38: 769-78.

5. Lazenby RA. Identification of sex from metacarpals: effect of side asymmetry. F Forensic Sci 1994; 39: 1188-94.

6. Zanella VP, Brown TM. Testing the validity of metacarpal use in sex assessment of human skeletal remains. F Forensic Sci 2003; 48: 17-20.

7. Case DT, Rose AH. Sex determination from hand and foot bone lengths. F Forensic Sci 2007; 52: 264-70.
8. Stojanowski CM. Sexing potential of fragmentary and pathological metacarpals. Am 7 Phys Anthropol 1999; 109: 245-52.

9. Lazenby RA. Population variation in second metacarpal sexual size dimorphism. Am 7 Phys Anthropol 2002; 118: 378-84.

10. Smith SL. Attribution of hand bones to sex and population groups. f Forensic Sci 1996; 41: 469-77.

11. Falsetti AB. Sex assessment from metacarpals of the human hand. 7 Forensic Sci 1995; 40: 774-6.

12. Barrio PA, Trancho GJ, Sánchez JA. Metacarpal sexual determination in a Spanish population. F Forensic Sci 2006; 51: 990-5.

13. Ruff CB, Scott WW, Liu AC. Articular and diaphyseal remodeling of the proximal femur with changes in mass in adults. Am 7 Phys Anthropol 1991; 86: 397-13.

14. Harma A, Karakaş HM. Determination of sex from the femur in Anatolian Caucasians: a digital radiological study. 7 Forensic Leg Med 2007; 14: 190-4.

Correspondence to: Assist. Prof. Banu Alıcıoğlu, MD Fatih Mah. 4. Cad. 43. Sok. Ziraatliler Sitesi B Blok No: 8 Edirne 22030 Turkey Phone: +90 284236 3089; GSM: +90 5323660070 e-mail: banualicioglu@trakya.edu.tr Conflict of interest statement: No conflicts declared. 